\title{
Guided Endodontics - An Update
}

\section{Karthika JR ${ }^{1 *}$, Lekshmy $S$ Devi² ${ }^{2}$ Lakshmi Aravind ${ }^{3}$ and Karthik $S^{4}$}

${ }^{1}$ Post Graduate, Department of Conservative Dentistry and Endodontics,

Sri Sankara Dental College, Varkala, Trivandrum, India

${ }^{2}$ Professor and Head, Department of Conservative Dentistry and Endodontics,

Sri Sankara Dental College, Varkala, Trivandrum, India

${ }^{3}$ Professor, Department of Conservative Dentistry and Endodontics, Sri Sankara

Dental College, Varkala, Trivandrum, India

${ }^{4}$ Assistant Professor, Department of Prosthodontics and Crown and Bridge,

Sri Sankara Dental College, Varkala, Trivandrum, India

*Corresponding Author: Karthika JR, Post Graduate, Department of Conservative

Dentistry and Endodontics, Sri Sankara Dental College, Varkala, Trivandrum, India.
Received: September 20, 2021

Published: October 21, 2021

(C) All rights are reserved by Karthika JR, et al.

\section{Abstract}

The concept of guided endodontics are used for access cavity preparation and Endodontic surgery, in order to achieve safer and predictable results. It offers highly predictable outcome and lower risk of iatrogenic damage. This technique may be a promising method for the endodontic or surgical treatment of complex cases.

Keywords: Static Guided Approach; Dynamic Guiding; Endodontic Guide; Sleeve; 3D Printing

\section{Introduction}

The concept of Minimally invasive Endodontics helps to preserve healthy coronal, cervical, and radicular tooth structure during endodontic treatment. It is based on the preservation of pericervical dentin (PCD). In conventional deroofing process, much of the PCD is lost, which reduces the fracture resistance of tooth. Guided endodontics helps in the preservation of PCD and offers the most conservative approach for cases with high difficulty level: calcified canals. The concept of Guided endodontics was introduced with the improvements in tomographic imaging and 3D printing technologies $[1,2]$. These computer-designed guides are used for access cavity preparation and endodontic surgery in order to achieve predictable and safe results [3,4]. Guided approach is also gaining popularity in endodontic surgery. 3D printed guide is used for osteotomy and root- end resection $[5,6]$.

\section{Types of guided approach}

Guided approach can be static/dynamic. In Static guided approach, the optical impression is merged with the CBCT for the design of a virtual drill path before the clinical procedure. In Dynamic guiding, CBCT data is combined with recordings of drill movement [7].

\section{D Guide Planning and Designing}

- $\quad$ CBCT Scan of involved teeth

- Surface scan

- Merging CBCT Scan and Surface scan. 
CBCT and surface scan were matched on radiographically visible structures like teeth of the patient. Software will create the virtual image of commercially available bur. Virtual copy is superimposed with the radiographically visible part of calcified canal. Different softwares are used like coDiagnostiX ${ }^{\mathrm{R}}$, 2Ingis ${ }^{\mathrm{R}}$ technology, Aceton ${ }^{\mathrm{R}}$ Imaging software $[8,9]$.
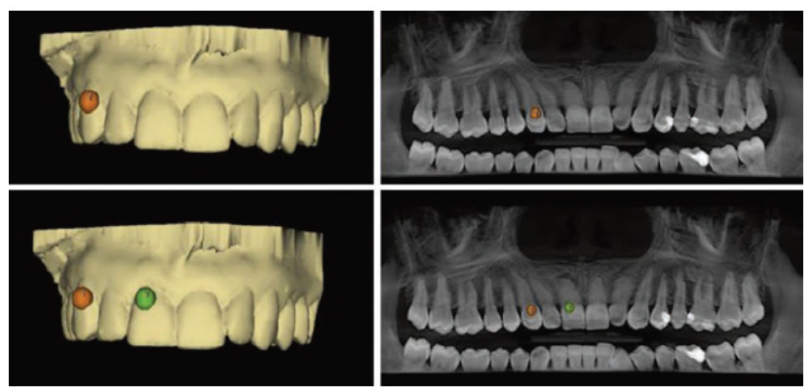

Figure 1: Superimposition of CBCT data and surface scan by marking three spots.

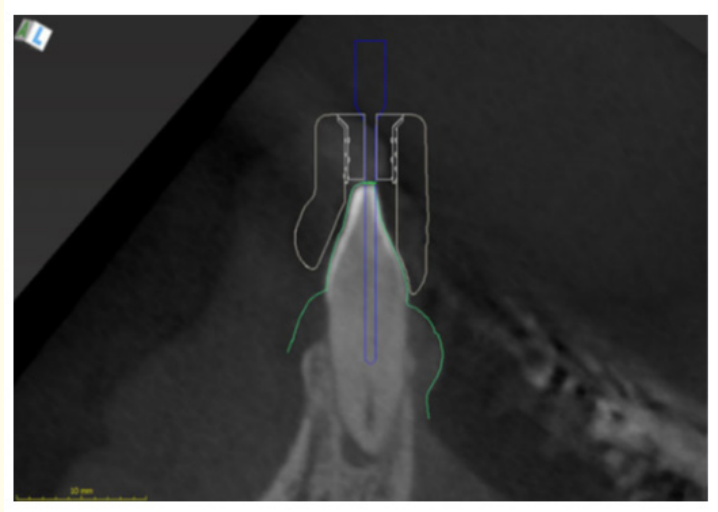

Figure 2: Virtual copy of drill is placed in such a way that the tip touches the radiographically visible part of the calcified root canal.

\section{Endoguide planning}

- Tracing the canal

- $\quad$ Sleeve selection.

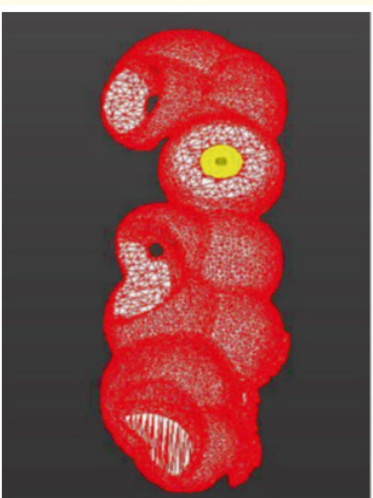

(a)

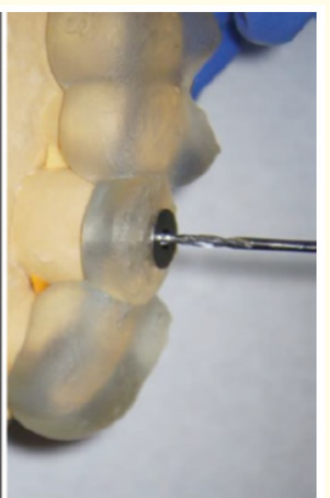

(b)
Figure 3: a. Meshwork of endodontic guide. b. Endodontic guide.

Guiding sleeve is added to the scan. The height of the sleeve should be $5-7 \mathrm{~mm}$ for the endodontic treatment [7].

\section{Documentation}

After creating a virtual template by applying designer tool of the software, virtual template will export as a Surface- tessellationlanguage (STL file) and produced by a 3D printer [10,11].

\section{Clinical procedure}

- Correct fit of the template will check on the patient

- Starting point of access preparation on tooth surface is marked with the colored resin

- After removing the guide, the entrance preparation should be done with a high speed bur

- Drilling directly on the dentinal surface with endoguide.

The rotational speed must be set to $10,000 \mathrm{rpm}$ and Microguided endodontic drill will be used to gain an access to the apical third of the root with pumping movements [7].

\section{Guided approach in Surgical endodontics}

3D Printed static aids: Applications for endodontic surgery. 


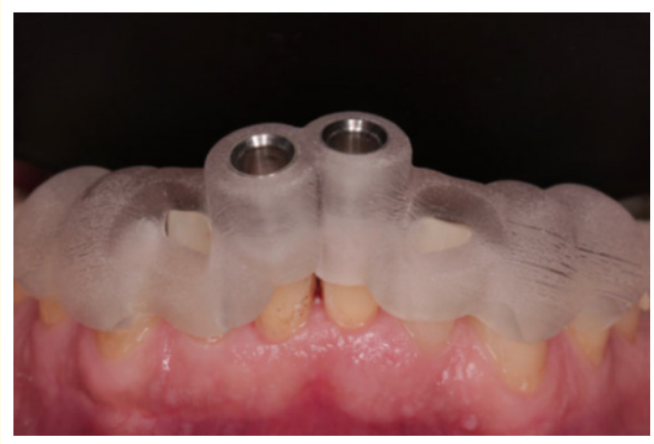

Figure 4: Well- fitting template in a correct position.

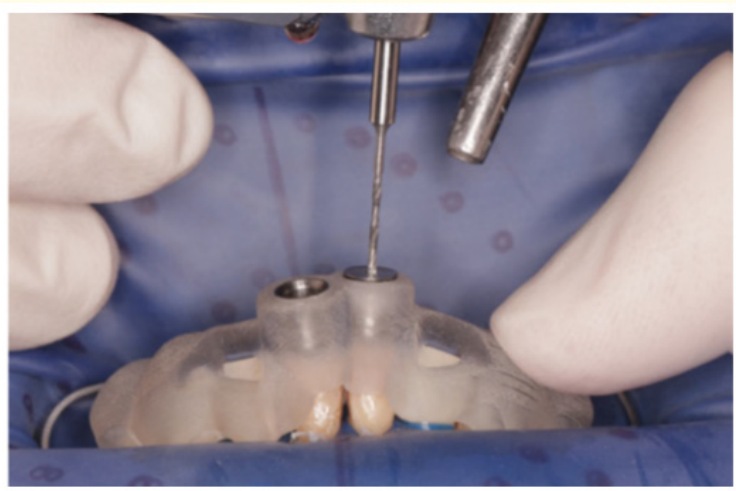

Figure 5: Access is gained to the calcified root canal using Guided endodontic technique.

\section{Nonguiding 3D printed template}

These are designed to define the surgical site $[12,13,16]$.

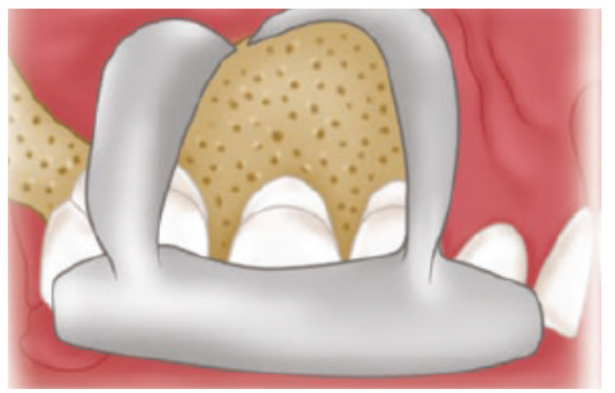

Figure 6: 3D printed endodontic surgical aid for soft tissue retraction [15].

\section{Pilot guide}

Pilot guide is used for the initial drilling and the bony cavity guides the subsequent drills.

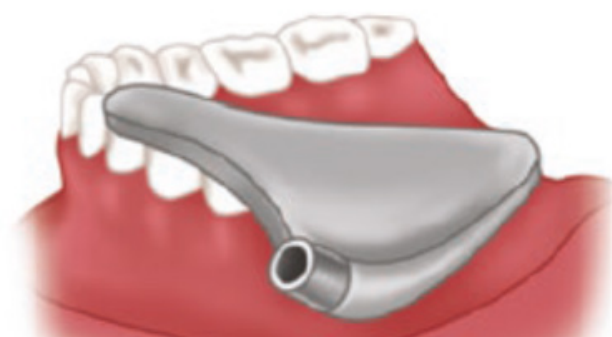

Figure 7

\section{Full guide for a Bone Trephine}

To perform osteotomy and root-end resection simultaneously.

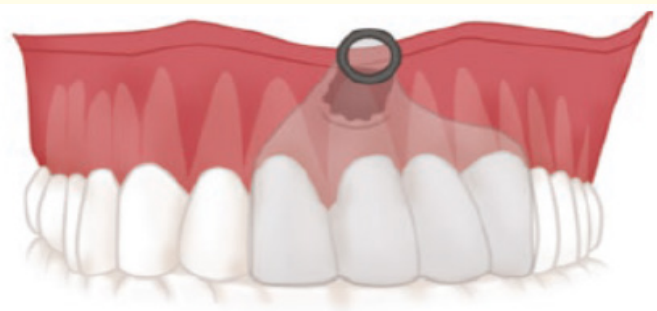

Figure 8: For bone trephine.

\section{D printed template for cortical preparation}

Helps to assess the exact site for root-end resection.

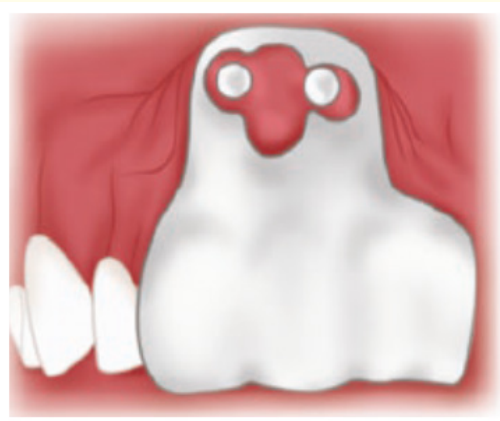

Figure 9 


\section{Advantages of guided approach}

- No need to use the Dental operating microscope

- $\quad$ Reduce the time of performing procedure [14].

Drawbacks of guided approach

- Canals with extremely narrow diameters may not be seen in the CBCT images as the voxel size is larger

- Static guide work only for the straight part of root canal

- Presence of metallic restorations/ filling of teeth leads to artifacts on radiograph [17-19].

\section{Conclusion}

Guided Endodontics offers safe and predictable method for locating calcified root canals. And it also offers lower risk of iatrogenic damage and it seems to be a highly promising technique. Targeted endodontic microsurgery provides complete and more appropriate root - end resection than traditional endodontic microsurgery.

\section{Bibliography}

1. Lara- Mendes., et al. "Guided endodontics as an alternative for the treatment of severly calcified root canals". Dental Press Endodontics 9.1 (2019): 15-20.

2. Kvinnsland., et al. "A Clinical and roentgenological study of 55 cases of root perforation". International Endodontic Journal 22 (1989): 75-84.

3. Van der Meer., et al. "3D Computer aided treatment planning in endodontics". Journal of Dentistry 45 (2016): 67-72.

4. Moreno- Rabie., et al. "Clinical applications, accuracy and limitations of guided endodontics: a systematic review". International Endodontic Journal 53 (2020): 214-231.

5. Andreasen., et al. "Occurrence of pulp canal obliteration after luxation injuries in the permanent dentition". Endodontics and Dental Traumatology 3 (1987): 103-115.

6. Fleig., et al. "Narrowing of the radicular pulp space in coronally restored teeth". Clinical Oral Investigations 21 (2017): 12511257.
7. Niraj Kinariwala Lakshman Samaranayake. “Guided Endodontics".

8. T Connert., et al. "Microguided Endodontics: a method to achieve minimally invasive access cavity preparation and root canal location in mandibular incisors using a novel computerguided technique". International Endodontic Journal 51 (2018): 247-255.

9. Zehnder., et al. "Guided Endodontics: accuracy of a novel method for guided access cavity preparation and root canal location". International Endodontic Journal 49 (2015): 966-972.

10. Krastl., et al. "Guided Endodontics: a novel treatment approach for teeth with pulp canal calcification and apical pathology". Dental Traumatology 32 (2016): 240-246.

11. Nayak., et al. "Computer- aided design-based guided endodontic: A novel approach for root canal access cavity preparation". Journal of Engineering in Medicine 8 (2018): 787-795.

12. Andreasen., et al. "Endodontic applications of 3D printing". International Endodontic Journal 51 (2018): 1005-1018.

13. Connert., et al. "Guided endodontics versus conventional access cavity preparation: a comparative study on substance loss using 3-dimensional- printed teeth". Journal of Endodontics 45 (2019): 327-331.

14. Byun., et al. "Endodontic treatment of an anomalous anterior tooth with the aid of a 3- dimensional printed physical tooth model". Journal of Endodontics 41 (2015): 961-965.

15. Giacomino., et al. "Targeted endodontic microsurgery: a novel approach to anatomically challenging scenarios using 3-dimensional- printed guides and trephine burs- a report of 3 cases". Journal of Endodontics 44 (2018): 671.

16. Ahn., et al. "Computer - aided design/computer - aided manufacturing - guided endodontic surgery: guided osteotomy and apex localization in a mandibular molar with a thick buccal bone plate". Journal of Endodontics 44 (2018): 665-670.

17. Patel., et al. "A novel method for soft tissue retraction during periapical surgery using 3D technology: a case report". International Endodontic Journal 50 (2017): 813-822. 
18. Cvek., et al. "Failures and healing in endodontically treated non-vital anterior teeth with posttraumatically reduced pulpal lumen". Acta Odontologica Scandinavica 40 (1982): 223-228.

19. Pinsky., et al. "Periapical surgery using CAD/ CAM guidance: preclinical results". Journal of Endodontics 33 (2007): 148-151.

Volume 5 Issue 11 November 2021

(C) All rights are reserved by Karthika JR., et al. 